\title{
The quotient of gamma functions by the psi function
}

\section{CRISTINEL MORTICI}

Valahia University of Târgovişte, Department of Mathematics, Bd. Unirii 18, 130082

Târgovişte, Romania

E-mails: cmortici@valahia.ro; cristinelmortici@yahoo.com

Abstract. The aim of this paper is to construct the asymptotic series of the ratio of gamma functions by Kershaw then to deduce some sharp estimates.

Mathematical subject classification: 33B15, 05A16, 26D15.

Key words: gamma function, digamma function, inequalities, asymptotic series, rate of convergence.

\section{Introduction}

The gamma function, defined for every $x>0$ by the formula

$$
\Gamma(x)=\int_{0}^{\infty} t^{x-1} e^{-t x} d t
$$

is of great importance in pure and applied mathematics and in consequence it attracted the attention of many authors. Some preoccupations are now directed to the problem of estimating the ratio

$$
Q(x, \beta)=\frac{\Gamma(x+\beta)}{\Gamma(x)} \quad(x>0,0<\beta<1)
$$

in terms of the digamma function $\psi=\Gamma^{\prime} / \Gamma$. The starting point of this problem can be considered the work of Gautschi [3], who proved

$$
Q(x, \beta)<\exp (\beta \psi(x+\beta)),
$$


for every $0<\beta<1$ and $x=1,2,3, \ldots$. This inequality (1.1) has found great interest, and several papers were subsequently published, for instance, by Erber [2], Kečkić and Vasić [4], Laforgia [6], Watson [26], or Zimering [27]. Furthermore, Lorch [9] found interesting connections of this inequality with the theory of ultraspherical polynomials.

Kershaw [5] improved (1.1), showing

$$
\exp (\beta \psi(x+\beta-1+\sqrt{1-\beta}))<Q(x, \beta)<\exp \left(\beta \psi\left(x+\frac{\beta}{2}\right)\right),
$$

for every $0<\beta<1$ and $x>1-\beta$. This inequality (1.2) is now known as the second Kershaw inequality.

Merkle [10] exploited some convexity arguments to improve the left-hand side of (1.2). By [10, Corollary 3], for every $0<\beta<1$ and $x>0$,

$$
\exp \left(\beta \frac{\psi(x+\beta)+\psi(x)}{2}\right)<Q(x, \beta) .
$$

Obviously, the main purpose of inequalities of type (1.1)-(1.3) is to obtain increasingly strong estimates of the ratio $Q(x, \beta)$, as $x$ approaches infinity. We consider that the natural way to attack such problems is to provide estimates of $Q(x, \beta)$ using asymptotic analysis theory. Although inequalities following by different convexity arguments are of intinsec beauty, they do not offer estimates to any expected accuracy.

In the first part of this paper we prove that the best approximation of the form

$$
Q(x, \beta) \approx \exp (\beta \psi(x+m)), \quad \text { as } x \rightarrow \infty
$$

where $m$ is a real parameter, is obtained indeed for $m=\beta / 2$.

In asymptotic theory, whenever an approximation of type (1.4) is given, there is a tendency to improve it by using an entire series of the form

$$
Q(x, \beta) \sim \exp \left(\beta \psi\left(x+\frac{\beta}{2}\right)\right) \exp \left(\sum_{j=1}^{\infty} \frac{a_{j}}{x^{j}}\right), \quad \text { as } x \rightarrow \infty .
$$

In general, such a series does not converge, but truncated of only a few terms, it provides approximations of any desired accuracy. 
One of the main result of this paper is the construction of the asymptotic expansion (1.5). We give a systematically way to find the coefficients $a_{j}$, while the first coefficients are the following

$$
\begin{aligned}
& a_{1}=0 \\
& a_{2}=-\frac{1}{24} \beta^{3} \\
& a_{3}=\frac{1}{24} \beta^{4}-\frac{1}{24} \beta^{3} \\
& a_{4}=\frac{1}{16} \beta^{4}-\frac{1}{48} \beta^{3}-\frac{11}{320} \beta^{5} \\
& a_{5}=\frac{1}{24} \beta^{4}-\frac{11}{160} \beta^{5}+\frac{13}{480} \beta^{6} \\
& a_{6}=\frac{1}{144} \beta^{3}-\frac{11}{192} \beta^{5}+\frac{13}{192} \beta^{6}-\frac{19}{896} \beta^{7}
\end{aligned}
$$

Numerical computations show that $Q(x, \beta)$ becomes closer to the righ-hand side of (1.2), for large values of $x$. In consequence, strong inequalities of the form

$$
\begin{gathered}
\exp \left(\beta \psi\left(x+\frac{\beta}{2}\right)\right) m_{1}(x, \beta)<Q(x, \beta) \\
<\exp \left(\beta \psi\left(x+\frac{\beta}{2}\right)\right) m_{2}(x, \beta)
\end{gathered}
$$

are obtained only if the quantities $m_{1}(x, \beta)$ and $m_{2}(x, \beta)$ becomes closer to 1 , as $x$ approaches infinity.

Motivated by these remarks, we use the first terms of expansion (1.5) to deduce the inequality of type (1.7) with

$$
\begin{aligned}
& m_{1}(x, \beta)=\exp \left(-\frac{\beta^{3}}{24 x^{2}}-\frac{\beta^{3}(1-\beta)}{24 x^{3}}\right), \\
& m_{2}(x, \beta)=\exp \left(-\frac{\beta^{3}}{24 x^{2}}\right),
\end{aligned}
$$

for every $0<\beta<1$ and sufficiently large integer $x$.

\section{The best approximation $Q(x, \beta) \approx \exp (\beta \psi(x+m))$}

We show in this section that the best approximation of the form (1.4) is obtained for $m=\beta / 2$. One method to estimate the accuracy of an approximation of 
type (1.4) is to introduce the relative error sequence $w_{n}$ by the relations

$$
Q(n, \beta)=\exp (\beta \psi(n+m)) \cdot e^{w_{n}}, \quad n=1,2,3, \ldots
$$

and to consider an approximation (1.4) better when $w_{n}$ faster converges to zero.

A powerful way to measure the rate of convergence is the following

Lemma 1. If $\left(\omega_{n}\right)_{n \geq 1}$ is convergent to zero and

$$
\lim _{n \rightarrow \infty} n^{k}\left(\omega_{n}-\omega_{n+1}\right)=l \in[-\infty, \infty],
$$

with $k>1$, then

$$
\lim _{n \rightarrow \infty} n^{k-1} \omega_{n}=\frac{l}{k-1} .
$$

This lemma was first used by Mortici [11]-[25] to construct asymptotic expansions and to improve some convergences. For complete proof of Lemma 1, see [11].

By (2.1), we have

$$
w_{n}=\ln Q(n, \beta)-\beta \psi(n+m)
$$

and as we are interested to compute a limit of the form (2.2), we write the difference

$$
w_{n}-w_{n+1}=\ln \frac{n}{n+\beta}+\frac{\beta}{n+m}
$$

as a power series into $n^{-1}$. Using a computer software, we get

$$
w_{n}-w_{n+1}=\left(\frac{\beta}{2}-m\right) \frac{\beta}{n^{2}}+\left(m^{2}-\frac{\beta^{2}}{3}\right) \frac{\beta}{n^{3}}+O\left(\frac{1}{n^{4}}\right) .
$$

Now we can apply Lemma 1 to establish the following

Theorem 1. (i) If $m \neq \beta / 2$, then $w_{n}$ converges as $n^{-1}$, since

$$
\lim _{n \rightarrow \infty} n w_{n}=\left(\frac{\beta}{2}-m\right) \beta \neq 0 .
$$

(ii) If $m=\beta / 2$, then $w_{n}$ converges as $n^{-2}$, since

$$
\lim _{n \rightarrow \infty} n^{2} w_{n}=-\frac{1}{24} \beta^{3} \neq 0 .
$$


Finally, the best approximation (1.4) appears when the maximum rate of convergence $n^{-2}$ of $w_{n}$ is attained, namely in case $m=\beta / 2$.

As a direct consequence of this fact, we mention that every other inequality of type $Q(x, \beta)<(>) \exp (\beta \psi(x+m))$, with $m \neq \beta / 2$ (largely studied in the literature) becomes weak for large values of $x$.

\section{The asymptotic expansion of $Q(x, \beta)$}

In this section, the main tool for constructing the asymptotic series (1.5) is the method introduced by Mortici in the recent paper [11].

Let us consider the approximation $f(n) \approx g(n)$, in the sense that the ratio of $f(n)$ and $g(n)$ tends to 1 as $n$ approaches infinity and assume that

$$
\ln \frac{f(n) g(n+1)}{g(n) f(n+1)}=\sum_{j=2}^{\infty} \frac{y_{j}}{n^{j}}
$$

Then the following asymptotic series holds

$$
f(n) \sim g(n) \exp \left(\sum_{j=1}^{\infty} \frac{a_{j}}{n^{j}}\right),
$$

where the coefficients $a_{j}$ are given by the infinite triangular system

$$
a_{1}-\left(\begin{array}{c}
j-1 \\
1
\end{array}\right) a_{2}+\cdots+(-1)^{j}\left(\begin{array}{l}
j-1 \\
j-2
\end{array}\right) a_{j-1}=(-1)^{j} y_{j}, \quad j \geq 2 .
$$

For proofs and other details, see [11].

Lemma 2. For

$$
f(n)=Q(n, \beta), \quad g(n)=\exp \left(\beta \psi\left(n+\frac{\beta}{2}\right)\right),
$$

we have

$$
\ln \frac{f(n) g(n+1)}{g(n) f(n+1)}=\sum_{j=2}^{\infty}(-1)^{j}\left(\frac{1}{j}-\frac{1}{2^{j-1}}\right) \beta^{j} \frac{1}{n^{j}}
$$


Proof. We have

$$
\begin{gathered}
\ln \frac{f(n) g(n+1)}{f(n+1) g(n)}=\frac{\beta}{n+\frac{\beta}{2}}+\ln \frac{n}{n+\beta} \\
=2\left(1-\frac{1}{1+\frac{\beta}{2 n}}\right)-\ln \left(1+\frac{\beta}{n}\right)=\sum_{j=2}^{\infty}(-1)^{j}\left(\frac{1}{j}-\frac{1}{2^{j-1}}\right) \beta^{j} \frac{1}{n^{j}} .
\end{gathered}
$$

Now, with

$$
y_{j}=(-1)^{j}\left(\frac{1}{j}-\frac{1}{2^{j-1}}\right) \beta^{j}, \quad j=2,3,4, \ldots,
$$

the first equations of the infinite system (3.1) becomes

$$
\left\{\begin{array}{l}
a_{1}=y_{2} \\
a_{1}-2 a_{2}=-y_{3} \\
a_{1}-3 a_{2}+3 a_{3}=y_{4} \\
a_{1}-4 a_{2}+6 a_{3}-4 a_{4}=-y_{5} \\
a_{1}-5 a_{2}+10 a_{3}-10 a_{4}+5 a_{5}=y_{6} \\
a_{1}-6 a_{2}+15 a_{3}-20 a_{4}+15 a_{5}-6 a_{6}=-y_{7}
\end{array}\right.
$$

with the solution (1.6).

\section{Sharp bounds of $Q(x, \beta)$}

As usually, we try to compare $Q(x, \beta)$ with approximations obtained by truncation the corresponding asymptotic expansion (1.5). More precisely, we prove that for large values of $x$, we have the following double inequality that improves much the Gautschi-Kershaw inequalities (1.1)-(1.3)

$$
\begin{aligned}
& \exp \left(\beta \psi\left(x+\frac{\beta}{2}\right)\right) \exp \left(-\frac{\beta^{3}}{24 x^{2}}-\frac{\beta^{3}(1-\beta)}{24 x^{3}}\right) \\
& <Q(x, \beta)<\exp \left(\beta \psi\left(x+\frac{\beta}{2}\right)\right) \exp \left(-\frac{\beta^{3}}{24 x^{2}}\right) .
\end{aligned}
$$

The left-hand side of (4.1) is valid for $\beta>\frac{30-4 \sqrt{15}}{33}$, while it reverses for $\beta<\frac{30-4 \sqrt{15}}{33}$. 
Theorem 2. There exists $n_{0}$ such that for every integer $x \geq n_{0}$ and $0<$ $\beta<1$, we have

$$
Q(x, \beta)<\exp \left(\beta \psi\left(x+\frac{\beta}{2}\right)\right) \exp \left(-\frac{\beta^{3}}{24 x^{2}}\right) .
$$

Proof. The sequence

$$
a_{n}=\beta \psi\left(n+\frac{\beta}{2}\right)-\frac{\beta^{3}}{24 n^{2}}-\ln Q(n, \beta)
$$

converges to zero. We prove that for some $n_{0}$, the sequence $\left(a_{n}\right)_{n \geq n_{0}}$ is strictly decreasing and consequently, $a_{n}>0$, for every $n \geq n_{0}$ and the theorem is proved.

In this sense, we have $a_{n+1}-a_{n}=f(n)$, where

$$
f(x)=\frac{\beta}{x+\frac{\beta}{2}}-\frac{\beta^{3}}{24}\left(\frac{1}{(x+1)^{2}}-\frac{1}{x^{2}}\right)-\ln \frac{x+\beta}{x} .
$$

We have

$$
f^{\prime}(x)=\frac{Q(x)}{12 \beta^{3} x^{3}(x+1)^{3}(x+\beta)(2 x+\beta)^{2}},
$$

where $Q(x)=24(1-\beta) x^{4}+\cdots-\beta^{3}$ is a fourth degree polynomial in $x$.

As the leading coefficient of $Q$ is positive, we have $Q>0$, on an interval of the form $\left[n_{0}, \infty\right)$ and consequently, $f$ is strictly increasing on $\left[n_{0}, \infty\right)$. But $f(\infty)=0$, so $f(x)<0$, for every $x \geq n_{0}$. Finally, the sequence $\left(a_{n}\right)_{n \geq n_{0}}$ is strictly decreasing, thus $a_{n}>0$ and the proof is completed.

Theorem 3. a) There exists $n_{1}$ such that for every integer $x \geq n_{1}$ and $\beta \in\left(0, \frac{30-4 \sqrt{15}}{33}\right)$, we have

$$
Q(x, \beta)<\exp \left(\beta \psi\left(x+\frac{\beta}{2}\right)\right) \exp \left(-\frac{\beta^{3}}{24 x^{2}}-\frac{\beta^{3}(1-\beta)}{24 x^{3}}\right) .
$$

b) There exists $n_{2}$ such that for every integer $x \geq n_{2}$ and $\beta \in\left(\frac{30-4 \sqrt{15}}{33}, 1\right)$, we have

$$
\exp \left(\beta \psi\left(x+\frac{\beta}{2}\right)\right) \exp \left(-\frac{\beta^{3}}{24 x^{2}}-\frac{\beta^{3}(1-\beta)}{24 x^{3}}\right)<Q(x, \beta) .
$$


Proof. The sequence

$$
b_{n}=\beta \psi\left(n+\frac{\beta}{2}\right)-\frac{\beta^{3}}{24 n^{2}}-\frac{\beta^{3}(1-\beta)}{24 n^{3}}-\ln Q(n, \beta)
$$

converges to zero. We prove that starting with some index, the sequence $b_{n}$ is strictly decreasing (respective increasing) and consequently, $b_{n}>0$, (respective $b_{n}<0$ ), and the theorem is proved.

In this sense, we have $b_{n+1}-b_{n}=g(n)$, where

$$
\begin{aligned}
g(x)= & \frac{\beta}{x+\frac{\beta}{2}}-\frac{\beta^{3}}{24}\left(\frac{1}{(x+1)^{2}}-\frac{1}{x^{2}}\right) \\
& -\frac{\beta^{3}(1-\beta)}{24}\left(\frac{1}{(x+1)^{3}}-\frac{1}{x^{3}}\right)-\ln \frac{x+\beta}{x} .
\end{aligned}
$$

We have

$$
g^{\prime}(x)=-\frac{P(x)}{24 \beta^{3} x^{4}(x+1)^{4}(x+\beta)(2 x+\beta)^{2}},
$$

where $P$ is a fifth degree polynomial in $x$,

$$
P(x)=\left(120 \beta-66 \beta^{2}-40\right) x^{5}+\cdots+\left(3 \beta^{3}-3 \beta^{4}\right) .
$$

Now, the leading coefficient of $P$ is negative if $\beta \in\left(0, \frac{30-4 \sqrt{15}}{33}\right)$, respective positive, if $\beta \in\left(\frac{30-4 \sqrt{15}}{33}, 1\right)$. In consequence, if $\beta \in\left(0, \frac{30-4 \sqrt{15}}{33}\right)$, then $P<0$ on an interval of the form $\left[n_{1}, \infty\right)$, and if $\beta \in\left(\frac{30-4 \sqrt{15}}{33}, 1\right)$, then $P>0$ on an interval of the form $\left[n_{2}, \infty\right)$.

As $g$ is proved to be strictly increasing on $\left[n_{1}, \infty\right.$ ) (respective strictly decreasing on $\left[n_{2}, \infty\right)$ ) and $g(\infty)=0$, it results that $g<0$ on $\left[n_{1}, \infty\right)$ (respective $g>0$ on $\left.\left[n_{2}, \infty\right)\right)$. Finally, if $\beta \in\left(0, \frac{30-4 \sqrt{15}}{33}\right)$, then $\left(b_{n}\right)_{n \geq n_{1}}$ is strictly decreasing, while if $\beta \in\left(\frac{30-4 \sqrt{15}}{33}, 1\right)$, then $\left(b_{n}\right)_{n \geq n_{2}}$ is strictly increasing and the theorem is proved.

\section{$5 Q(x, 1 / 2)$ and the Wallis ratio}

The general results obtained in the previous sections can be extended, for particular cases of $\beta \in(0,1)$, if we think that the polynomials involved in (4.2) and (4.5) becomes simpler. 
More precisely, the privileged value $\beta=1 / 2$ is of great interest, since $Q(x, 1 / 2)$ is close related with the Wallis ratio $\frac{\Gamma(x+1)}{\Gamma\left(x+\frac{1}{2}\right)}$ and with the Wallis sequence

$$
W_{n}=\frac{1}{2 n+1}\left(\frac{1 \cdot 3 \cdot 5 \cdot \ldots \cdot(2 n-1)}{2 \cdot 4 \cdot 6 \cdot \ldots \cdot 2 n}\right)^{2} .
$$

Moreover, the Wallis ratio appears in the problem of estimating the volume and the surface area of the unit ball in $\mathbb{R}^{n}$. See, e.g., [1, p. 258, Relation 6.1.49], or [17].

Gautschi [3] proved

$$
\sqrt{n}<\frac{\Gamma(n+1)}{\Gamma\left(n+\frac{1}{2}\right)}<\sqrt{n+1},
$$

then Kazarinoff [8, pp. 47-48 and pp. 65-67] showed

$$
\sqrt{n+\frac{1}{4}}<\frac{\Gamma(n+1)}{\Gamma\left(n+\frac{1}{2}\right)}<\sqrt{n+\frac{1}{2}} .
$$

We obtain here sharp estimates for $Q(x, 1 / 2)$, using the asymptotic expansion (1.5), which becomes

$$
\begin{gathered}
Q\left(x, \frac{1}{2}\right) \sim \exp \left(\frac{1}{2} \psi\left(x+\frac{1}{4}\right)\right) \\
\times \exp \left(-\frac{1}{192 x^{2}}-\frac{1}{384 x^{3}}+\frac{7}{30720 x^{4}}+\frac{9}{10240 x^{5}}-\frac{31}{1032192 x^{6}}+\cdots\right) .
\end{gathered}
$$

The double inequality (4.1) becomes

$$
\begin{gathered}
\exp \left(\frac{1}{2} \psi\left(x+\frac{1}{4}\right)\right) \exp \left(-\frac{1}{192 x^{2}}-\frac{1}{384 x^{3}}\right) \\
<Q\left(x, \frac{1}{2}\right)<\exp \left(\frac{1}{2} \psi\left(x+\frac{1}{4}\right)\right) \exp \left(-\frac{1}{192 x^{2}}\right),
\end{gathered}
$$

but we able now to prove the following stronger result.

Theorem 4. For every positive integer $x$, we have

$$
\begin{gathered}
\exp \left(\frac{1}{2} \psi\left(x+\frac{1}{4}\right)\right) \exp \left(-\frac{1}{192 x^{2}}-\frac{1}{384 x^{3}}+\frac{7}{30720 x^{4}}\right) \\
<Q\left(x, \frac{1}{2}\right)<\exp \left(\frac{1}{2} \psi\left(x+\frac{1}{4}\right)\right) \\
\quad \times \exp \left(-\frac{1}{192 x^{2}}-\frac{1}{384 x^{3}}+\frac{7}{30720 x^{4}}+\frac{9}{10240 x^{5}}\right) .
\end{gathered}
$$


Proof. We prove that the convergent to zero sequences

$$
u_{n}=\frac{1}{2} \psi\left(n+\frac{1}{4}\right)-\frac{1}{192 n^{2}}-\frac{1}{384 n^{3}}+\frac{7}{30720 n^{4}}-\ln Q\left(n, \frac{1}{2}\right)
$$

and

$$
\begin{aligned}
v_{n}= & \ln Q\left(n, \frac{1}{2}\right)-\frac{1}{2} \psi\left(n+\frac{1}{4}\right) \frac{1}{192 n^{2}}+\frac{1}{384 n^{3}} \\
& -\frac{7}{30720 n^{4}}-\frac{9}{10240 n^{5}}
\end{aligned}
$$

are strictly increasing, thus $u_{n}<0$ and $v_{n}<0$.

Let $u_{n+1}-u_{n}=s(n), v_{n+1}-v_{n}=t(n)$, where

$$
\begin{aligned}
s(x)= & \frac{1}{2 x+\frac{1}{2}}-\frac{1}{192}\left(\frac{1}{(x+1)^{2}}-\frac{1}{x^{2}}\right)-\frac{1}{384}\left(\frac{1}{(x+1)^{3}}-\frac{1}{x^{3}}\right) \\
& +\frac{7}{30720}\left(\frac{1}{(x+1)^{4}}-\frac{1}{x^{4}}\right)-\ln \frac{x+\frac{1}{2}}{x}
\end{aligned}
$$

and

$$
\begin{aligned}
t(x)= & \ln \frac{x+\frac{1}{2}}{x}-\frac{1}{2 x+\frac{1}{2}}+\frac{1}{192}\left(\frac{1}{(x+1)^{2}}-\frac{1}{x^{2}}\right) \\
& +\frac{1}{384}\left(\frac{1}{(x+1)^{3}}-\frac{1}{x^{3}}\right)-\frac{7}{30720}\left(\frac{1}{(x+1)^{4}}-\frac{1}{x^{4}}\right) \\
& -\frac{9}{10240}\left(\frac{1}{(x+1)^{5}}-\frac{1}{x^{5}}\right) .
\end{aligned}
$$

The functions $s(x)$ and $t(x)$ are strictly decreasing on $[1, \infty)$, since

$$
s^{\prime}(x)=-\frac{6480 x^{6}+15890 x^{5}+13705 x^{4}+4606 x^{3}+336 x^{2}-45 x-7}{7680 x^{5}(x+1)^{5}(2 x+1)(4 x+1)^{2}}
$$

and

$$
t^{\prime}(x)=-\frac{135+2188 x+14653 x^{2}+52026 x^{3}+99977 x^{4}+99016 x^{5}+40920 x^{6}+1240 x^{7}}{30720 x^{6}(x+1)^{6}(2 x+1)(4 x+1)^{2}} .
$$

Moreover, $s(\infty)=t(\infty)=0$, so $s>0$ and $t>0$ on [1, $\infty$ ). Finally, $u_{n}$ and $v_{n}$ are strictly increasing and the theorem is proved. 
Acknowledgments. This work was supported by a grant of the Romanian National Authority for Scientific Research, CNCS - UEFISCDI, project number PN-II-ID-PCE-2011-3-0087.

\section{REFERENCES}

[1] M. Abramowitz and I.A. Stegun, Handbook of Mathematical Functions with Formulas, Graphs, and Mathematical Tables. National Bureau of Standards, Applied Mathematical Series, 55, $9^{\text {th }}$ printing, Dover, New York (1972).

[2] T. Erber, The gamma function inequalities of Gurland and Gautschi. Skanid. Aktuarietidskr., (1960, 1961), 27-28.

[3] W. Gautschi, Some elementary inequalities relating to the gamma and incomplete gamma function. J. Math. Phys., 38 (1959), 77-81.

[4] J.D. Kečkić and P.M. Vasić, Some inequalities for the gamma function. Publ. Inst. Math. (Beograd) (N.S.), 11 (1971), 107-114.

[5] D. Kershaw, Some extensions of W. Gautschi's inequalities for the gamma function. Math. Comp., 41 (1983), 607-611.

[6] A. Laforgia, Further inequalities for the gamma function. Math. Comp., 42 (1984), 597-600.

[7] C. Giordano, A. Laforgia and J. Pečarić, Unified treatment of Gautschi-Kershaw type inequalities for the gamma function. J. Comput. Appl. Math., 99 (1998), $167-175$.

[8] N.D. Kazarinoff, Analytic Inequalities, Holt, Rhinehart and Winston, New York, (1961).

[9] L. Lorch, Inequalities for ultraspherical polynomials and the gamma function. J. Approx. Theory, 40 (1984), 115-120.

[10] M. Merkle, Convexity, Schur-convexity and bounds for the Gamma function involving the Digamma function. Rocky Mountain J. Math., 28 (1998), 1053-1066.

[11] C. Mortici, Product approximations via asymptotic integration. Amer. Math. Monthly, 117(5) (2010), 434-441.

[12] C. Mortici, New approximations of the gamma function in terms of the digamma function. Appl. Math. Lett., 23(1) (2010), 97-100.

[13] C. Mortici, An ultimate extremely accurate formula for approximation of the factorial function. Arch. Math. (Basel), 93(1) (2009), 37-45. 
[14] C. Mortici, New sharp bounds for gamma and digamma functions. An. Şt. Univ. A.I. Cuza Iaşi, Ser. Mat., 57(1) (2011).

[15] C. Mortici, Completely monotonic functions associated with gamma function and applications. Carpathian J. Math., 25(2) (2009), 186-191.

[16] C. Mortici, The proof of Muqattash-Yahdi conjecture. Math. Comput. Modelling, 51(9-10) (2010), 1154-1159.

[17] C. Mortici, Monotonicity properties of the volume of the unit ball in $\mathbb{R}^{n}$. Optimization Lett., 4(3) (2010), 457-464.

[18] C. Mortici, Sharp inequalities related to Gosper's formula. C.R. Math. Acad. Sci. Paris, 348(3-4) (2010), 137-140.

[19] C. Mortici, A class of integral approximations for the factorial function. Comput. Math. Appl., 59(6) (2010), 2053-2058.

[20] C. Mortici, Best estimates of the generalized Stirling formula. Appl. Math. Comput., 215(11) (2010), 4044-4048.

[21] C. Mortici, Very accurate estimates of the polygamma functions. Asympt. Anal., 68(3) (2010), 125-134.

[22] C. Mortici, Improved convergence towards generalized Euler-Mascheroni constant. Appl. Math. Comput., 215(9) (2010), 3443-3448.

[23] C. Mortici, A coincidence degree for bifurcation problems. Nonlin. Anal., 53(5) (2003), 715-721.

[24] C. Mortici, A quicker convergence toward the $\gamma$ constant with the logarithm term involving the constant e. Carpathian J. Math., 26(1) (2010), 86-91.

[25] C. Mortici, Optimizing the rate of convergence in some new classes of sequences convergent to Euler's constant. Anal. Appl. (Singap.), 8(1) (2010), 99-107.

[26] G.N. Watson, A note on gamma functions. Proc. Edinburgh Math. Soc., 11(2) (1958/59), Edinburgh Math. Notes 41 (1959), 7-9.

[27] S. Zimering, On a Mercerian theorem and its application to the equiconvergence of Cesaro and Riesz transforms. Publ. Inst. Math. (Beograd) (N.S.), 1 (1961), 83-91. 